\title{
Selection of a HER2/Neu Specific scfv by Ribosome Display for Targeting of HER2/Neu Positive Cancers
}

\section{Fatemeh Salimi ${ }^{1, *}$}

${ }^{1}$ Department of medical biotechnology, Faculty of medical sciences, Tarbiat Modares University, Tehran, Iran

${ }^{*}$ Corresponding Author: Fatemeh Salimi, Department of medical biotechnology, Faculty of medical sciences, Tarbiat Modares University, Tehran, Iran. E-mail:

DOI: $10.21859 /$ mci-supp-54 F.salimi@modares.ac.ir

Keywords: HER2/Neu

SCFV

Ribosome Display

\begin{abstract}
Introduction Targeting cancer cells through specific tumor markers is an important step in cancer diagnosis and treatment. Development of a powerful method to select high affinity binders for cancer cell surface receptors is of most importance. Human epithelial growth factor receptor (HER-2) is one of these markers overexpressed in many cancer types. Ribosome display is a method that acts like affinity maturation of natural immune system to produce binders like SCFVs against many kinds of cancer antigens. scfv antibodies have been used extensively for targeting surface markers of cancer cells. In this study, we constructed a murine single chain antibody library and then select a high affinity scfv against HER2/Neu by ribosome display method.
\end{abstract}

Materials and Methods: Briefly, we immunized four female BALB/C mouse with HER2/ Neu over-expressing cell line. RNA was extracted from spleen and the library were constructed with assembly of amplified Heavy and Kappa chains of antibody with a (G4S) 3 flexible linker using overlap-extension PCR. Antibody/ribosome/mRNA complexes produced from In-vitro transcribed/translated DNA library by TNT/quick system, were used for selection of binders in microtiter wells coated with Her2/Neu antigen. After washing the plates, bound complexes were eluted, RNA receovered and reverse transcribed and were used for amplification with library primers in PCR. Resultant sequences were cloned in pET28a vector and some of the selected SCFVs were expressed in BL21 host. Proteins were analysed by SDS/PAGE. They are extracted from bacterial crude extract and affinity of them was measured by ELISA.

Results: Our results show the good binding affinity of selected single chain antibody to the extracellular domians of HER2/Neu.

Conclusions: Accordingly, Ribosome display may be a robust method to select an antibody against any other cancer antigens in a rapid and relieble way compared to the other selection methods like phage display. 